\title{
Investigation of a Magnetic-Type Waterproof Oil Spill Stop Device for Ships
}

\section{Chong-Ju Chae}

Korea Institute of Maritime and Fisheries Technology (KIMFT), 367 Haeyang-ro, Busan 49111, Korea; katheshe76@naver.com; Tel.: +82-10-4548-0322

Received: 17 July 2020; Accepted: 18 August 2020; Published: 20 August 2020

\begin{abstract}
If a ship's hull or tank breaks, the ship may sink, or oil spills can cause enormous damage to the environment. If the ship is equipped with a capable, cost-effective oil or liquid flow stop emergency device, casualties and marine pollution could be reduced. Many magnetic-type liquid spill stop emergency devices developed since 1904 have limitations, such as difficulties with installation and impossibility of use during sailing. This study demonstrated the applicability of a magnetic-type liquid spill stop emergency device through tests for water pressure and leakage, attachment, magnetic fields, and the generation of sparks. Results showed that the device can be applied to the ship's side hull and bottom with a specified minimum diameter at a pressure depth of $1.0 \mathrm{~kg} / \mathrm{cm}^{2}$ while sailing at a speed of $18 \mathrm{kts}(9.26 \mathrm{~m} / \mathrm{s})$. If the distance from the device was at least approximately $750 \mathrm{~mm}$, the magnetic field had no effect, and there was no risk of explosion due to sparks. A cost-benefit analysis based on the International Maritime Organization-approved guidelines for formal safety assessment confirmed the cost effectiveness of the device. This experimental study confirmed that the magnetic liquid stop emergency device is generally applicable to a ship's hull.
\end{abstract}

Keywords: magnetic liquid stop device; oil spill prevention; oil spill stop device; FSA; cost-benefit technology; risk control options

\section{Introduction}

Oil spills caused by collisions and the stranding of ships led to improvements in the International Maritime Organization (IMO) regulatory framework and eventually in maritime safety and operation [1]. Hull failure was one of the major causes of oil spills from ships during 1974-2016 [2]. Clearly, an oil spill from a vessel is a critical maritime accident. The groundings of "Torrey Canyon" in 1967 and "Argo Merchant" in 1976 led to the creation of tanker fire safety regulations: MARPOL 1973, STCW 1978, and SOLAS 1974. Furthermore, the grounding of "Amoco Cadiz" in 1978 led to the creation of the Protocol of 1978 of MARPOL [3]. The "Exxon Valdez" disaster in 1989 led to introduction of the double-hull tanker [4]. The "Erika" disaster in 1999 contributed to the revision of MARPOL 73/78 Regulation 13G, which accelerated the phase-out of single hull tankers [5]. After the "Prestige" accident in 2002, the European Union fast-tracked not only single hull tanker phase-out but also the carriage of heavy grade oils in double hull tankers. The "Hebei Spirit" oil spill accident in 2007 caused a loss of 10.900 tons of cargo from five punctures in the hull [6]. A voluntary nationwide clean-up was carried out to remove the oil spilled from the accident. The number of people involved in the clean-up work exceeded 1 million in the month of the accident [7]. In the case of vessels transporting cargo, if an opening or breach in a hull, tank, or other containment structure occurs as a result of an accident, the internal cargo is susceptible to accidental discharge from the vessel into the environment, especially if the cargo is a liquid such as oil [8]. In addition, the damage sustained by vessels can have adverse effects on the stability of the vessel. Since the location and extent of the damage is often unknown at the time of an accident, the operator of the vessel cannot make well-informed decisions about the best 
strategy for preventing the vessel from danger or about the extent of the potential threat of accidental discharge of cargo [8]. Nevertheless, oil pollution caused by ship collisions and stranding continues to occur, and tanker vessels are particularly at risk. According to Vasconcellos et al. (2018), half of the world's oil is transported by vessels sailing over oceans [9]. The European Space Agency has stated that more than 4.5 million tons of oil are spilt per year, where $45 \%$ of the amount is due to operative discharge from ships [10]. For example, approximately 304.700 tons of oil were spilt from oil tankers and ships in the Mediterranean Sea between 1977 and 2003 [11], and approximately 5.86 million tons of oil were lost globally as a result of tanker accidents from 1970 to 2019 [12,13].

In 2002, a formal safety assessment (FSA) guideline for a rational decision-making system was developed to amend the IMO conventions [14]. According to this guideline, FSAs were conducted for various vessel types [15-18]. In particular, the IMO presented risk control options (RCOs) to cope with identified risks by FSAs for the safe operation of tanker vessels, to protect the marine environment from oil leakage-related risks. The FSA for tankers in 2008 presented RCOs such as the installation of steering gear redundancy, installation of navigational sonar, design modifications to reduce collision, installation of proximity and speed sensors in the terminal, installation of dual fuel supply lines, installation of hull stress and a fatigue monitoring system, and installation of an electronic chart display and information system [18]. However, some of the RCOs presented by the FSA were not applied. Among the many reasons for this, cost considerations were of the utmost importance [19]. The RCOs in the FSA can be considered to be cost-effective because they were selected through cost-benefit analysis. Nevertheless, some items require high additional costs for actual application, thus presenting a dilemma regarding compliance with the results of the FSA [19]. Thus, enforcing these conditions through the IMO convention can pose a considerable economic burden for ship owners. In particular, suggestions for tanker design changes such as the subdivision of cargo tank compartments, double hull height expansion, and side tank width expansion have limitations in actual applications in terms of cost, despite their proven effectiveness [18]. Therefore, when RCOs are proposed, those with a rational cost acceptable to ship owners need to be considered as well. In particular, cost-effective suggestions should be made when developing systems or applying regulations for the prevention of flooding due to ship accidents and the prevention of pollution resulting from oil spills.

If a hole is present in a ship's hull, a few techniques are available to prevent the leakage of liquid from the hole, such as using a wood plug, pad, or tape band, and underwater welding. Numerous devices have been suggested by prior studies directed toward this problem. Of these, many proposed a patch or curtain that is sealed on all sides of the rupture, such as Frisbie U.S. Pat. No. 351,971 [20], Weihe U.S. Pat. No. 381,302 [21], Richardson U.S. Pat. No. 385,572 [22], Buce U.S. Pat. No. 3,669,055 [23], and McDuff U.S. Pat. No. 4,712,502 [24]. However, these demonstrate unnecessary complexity and/or limited effectiveness, as well as difficulty in forming a seal at the bottom of the vessel [25].

Oil spill stop devices using magnetic force are among the methods developed long ago to prevent the inflow or outflow of liquid (such as seawater and oil) in the event of an oil spill in a ship. In 1904, L. Kruger first introduced an oil spill stop device for ships using magnets with a magnetic curtain for covering leaks in ships [26]. Later, Buce developed an apparatus and a method for sealing a vessel opening to prevent the leakage of liquids, such as oil, from the hull [23]. Johnson proposed a spill prevention device through oil spill control [25]; Uri developed an oil spill stop device using electromagnetic bars in a patch system for ship hulls [8]. Williams devised a magnetic ship hull patch [27]. Prysner developed an oil spill prevention blanket [28]; unlike the existing methods, it has the ability to flexibly block the oil spill region. Cunningham et al. developed an apparatus to attach a remote-controlled device for sealing openings in the hulls of vessels using electromagnets to move a patch to the position of the oil leak and attach it using rivets [29]. Furthermore, Cunningham et al. developed a remotely operated unmanned vehicle using electromagnets that could be operated to block an oil spill from a broken hull. Equipped with an imaging system to monitor the surrounding conditions, this vehicle can block oil leaks, thus preventing the leakage of cargo or the inflow of liquid [29]. Del Raso installed a novel collapsible bladder device on the cargo tank of an oil tanker to 
block possible oil leaks [30]. Moon et al. (2013) developed a liquid spill stopper using magnetic force and investigated its usability through a pressure experiment and separation experiments in relation to the ship speed with the device attached to a ship's hull [31]. Recently, small oil spill stopping devices using magnetic force, such as Miko Plaster, have been developed and are being applied to stop the inflow and outflow of oil through ship hull punctures [32,33].

Since 1904, magnetic-type oil spill stopping devices have been developed and patented in various forms; however, they have several limitations when put into actual use. The device developed by Kruger was designed to block oil spills using a magnetic roller curtain, but its installation was inconvenient, and the magnetic force applied was weak [26]. Another disadvantage was that it could not be used when the outflow pressure was high or when the puncture was at the bottom of the hull [26]. Moreover, it required an additional mechanism for fixing the device to the hull puncture, thus preventing its use in response to emergencies. Another device required a considerable amount of time for installation [25]. Furthermore, one device could not effectively stop leaks during sailing [28]. Another magnetic oil spill stopping device had limited applications with uneven surfaces [27]. Moon et al. conducted a water pressure test using a magnetic oil spill stopping device and conducted an experiment to assess the detachment of the device during sailing. The device was deformed by the water pressure because a rubber pad was used in the water pressure test. Furthermore, the test investigated the attachment of the device only to the ship's sheer side and could not verify attachment to the bottom of the ship [31]. The brackets (Miko Plaster) developed and employed by MIKO Marine are considered to be suitable for preventing oil spills. However, they seem to exbibit limitations when applied to liquid outflows underwater; hence, they are applied locally only when resistance in low [33]. The objectives of this study are to investigate the applicability and effectiveness of a magnetic-type, waterproof oil spill stop device (hereinafter referred to as "magnetic liquid stop device") and to perform a cost-benefit analysis to evaluate it as an RCO to reduce the risk of a ship sinking or an oil spill due to hull failure. To meet these objectives, a magnetic liquid stop device was used developed to demonstrate the effective application of the device in a rapid response to prevent marine pollution due to hull damage and reduce the risk of losing the seaworthiness of the ship due to flooding.

Then, the device was assessed for its feasibility, including a cost-benefit analysis, by applying and testing it to a ship. This study experimentally demonstrated that the magnetic liquid stop device can be applied effectively and rapidly to prevent marine pollution due to ship damage and reduce the risk of losing seaworthiness due to flooding. In addition, to improve upon the previously developed devices and related research, this study applied a magnetic liquid stop device that can easily be installed in the event of a puncture, can be applied to punctures on the ship's bottom, and is usable even on a round surface and during sailing. The cost-benefit analysis assessed the device as cost effective to be considered as an RCO. To this end, this study was conducted based on the method and procedure in Figure 1.

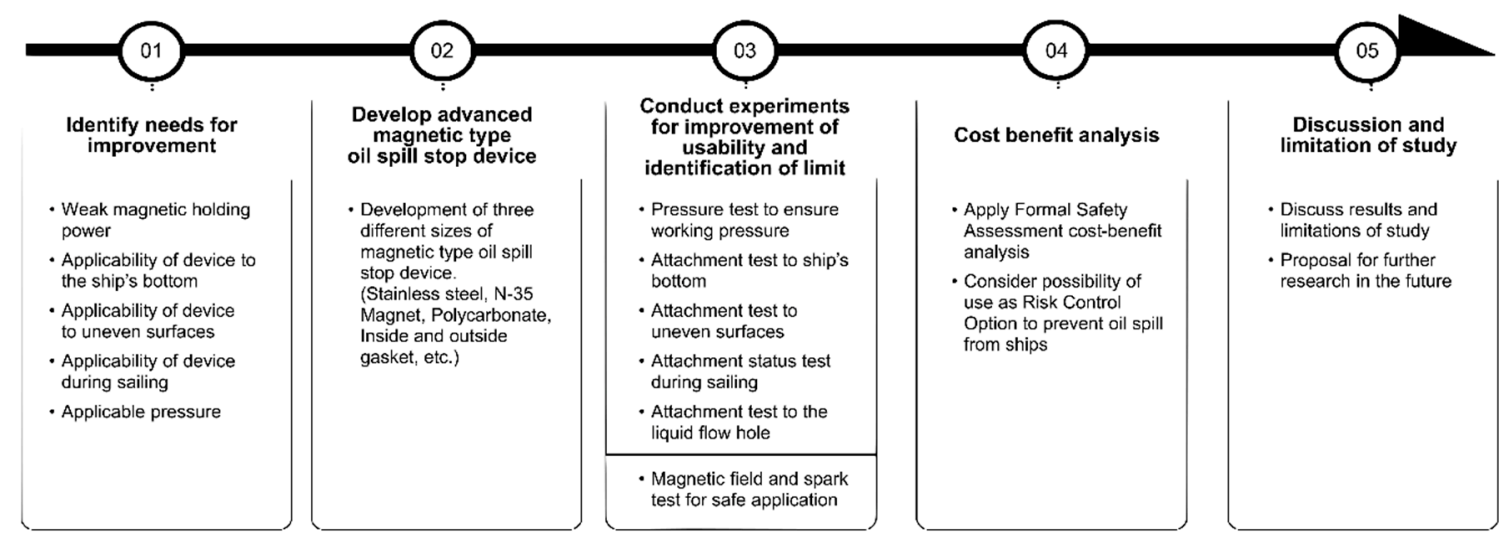

Figure 1. Method and procedure of this study. 
Based on the literature review, the aspect of magnetic-type oil spill stop devices that need improvement were identified. The device was developed by incorporating the improvement, and it was verified experimentally. The applicability of the device and limitations of the experiment were identified, and cost-benefit analysis of the device was performed. Based on this, the limitations of the device and future research directions are presented. This scope of this study is limited to the method and procedure in Figure 1.

\section{Materials and Methods}

A search for procedures and standards to test the usability of magnetic liquid stop devices yielded no results. Therefore, this study used the challenges identified in past studies to design the experimental study, which tests the installation of the device on a round surface on the ship's bottom and during sailing. In addition, a magnetic field test and a spark test were performed. The tests were performed in the presence of DNV-GL (classification society) surveyors to enhance the reliability of the tests, which was secured through test reports.

\subsection{Design Criteria for Magnetic Liquid Stop Device}

The magnetic liquid stop device was developed to be attached to the outside or inside of the hull in the event of a puncture in the hull, to stop the inflow of seawater in an emergency, prevent the sinking of ships, or prevent marine pollution by outflow of the oil. An Nd-Fe-B magnet (N-35 magnet), which is a powerful magnet that comprises rare earth elements, was used in the magnetic liquid stop device. Although the N-35 magnet is powerful, it is easily corroded by seawater $[34,35]$ and carries the risk of creating sparks when it hits the hull directly. Therefore, the N-35 magnet was coated with rubber to compensate for corrosion and sparks. Furthermore, resin-based elastic foam was applied to the outside and inside of the magnet to prevent the inflow of seawater and the outflow of oil during the use of the magnet. Flexible and elastic rubber and polyurethane resin with high tensile strength and low hardness (HB and HR values) were used in the main body of the magnetic liquid stop device for realizing high usability and resilience when applied to round surfaces. Additionally, stainless steel was used in the main body. A magnet holding power of $\geq 20 \mathrm{kgf} / \mathrm{ea}$ (each magnet) was obtained; the magnet's magnetic force reduction (aging change) is less than $1.7 \%$ in 10 years. The inside gasket was fabricated to provide an adhesive strength of $\geq 50 \mathrm{kgf} / \mathrm{cm}^{2}$ with stainless steel. In addition, it was designed to have excellent salt and corrosion resistance to prevent functional damage even after storage and use for a long time. The magnetic liquid stop device was developed in three sizes, considering application to different puncture sizes, convenience of mobility, and convenience of installation. The device was produced transparently, and a steel plate was inserted between the polyurethane resins to enhance the ability of the device to maintain its shape. The detailed specifications of the device are outlined in Table 1, and Figure 2 shows the products to be applied in the investigation. 
Table 1. Specifications of size, material of main body((cold rolled stainless steel plates, KSD), (stainless steel, STS), (Brinell Hardness, HB), (Rockwell Hardness, HR)), magnet type and size(Neodymium, (magnetic flux density, Br), (coercive force, bHc), (intrinsic coercive force, $\mathrm{iHc}$ ), (max energy, $\mathrm{BH})$ ), type of inside and out side of gasket of the magnetic liquid stop devices developed (2017) [36,37].

\begin{tabular}{|c|c|c|c|}
\hline & Large (WPP-L) & Medium (WPP-M) & Small (WPP-S) \\
\hline Width × length (mm) & $933 \times 638$ & $657 \times 431$ & $381 \times 293$ \\
\hline Number of magnets (ea) & 42 & 28 & 16 \\
\hline Spring plate size $(\mathrm{mm})$ & $905 \times 610$ & $627 \times 401$ & $351 \times 263$ \\
\hline Weight $(\mathrm{kg})$ & 16.5 & 10 & 4.5 \\
\hline Main body $\backslash$ & \multicolumn{3}{|c|}{$\begin{array}{l}\text { Stainless steel (KSD 3698, STS 301) } \\
\text { Tensile strength: } 500 \mathrm{~N} / \mathrm{mm}^{2} \\
\text { Hardness: HB } \leq 207, \mathrm{HR} \leq 95\end{array}$} \\
\hline $\begin{array}{c}\text { Magnet } \\
\text { (size: } 64 \mathrm{~mm} \times 39 \mathrm{~mm} \times 10 \mathrm{~mm})\end{array}$ & $\begin{array}{c}\text { Materials: Nd-Fe-B magnet } \\
\text { (N-35) } \\
\text { Br: } \geq 1170 \\
\text { bHc: } \geq 836 \mathrm{KA} / \mathrm{m} \\
\text { iHc: } \geq 955 \mathrm{KA} / \mathrm{m} \\
\text { (BH): } \geq 263 \mathrm{KJ} / \mathrm{m}^{3} \\
\text { Holding power: } \geq 20 \mathrm{Kgf} / \mathrm{ea}\end{array}$ & \begin{tabular}{|c|}
$\quad \oplus$ \\
\end{tabular} & $\frac{10}{\underbrace{\omega}_{\omega}}$ \\
\hline Inside gaskets & \multicolumn{3}{|c|}{$\begin{array}{c}\text { Hardness: } 82 \pm 2 \text { Shore A } \\
\text { Tensile strength: } 330 \pm 70 \mathrm{Kgf} / \mathrm{cm}^{2} \\
\text { Elongation at break: } 500 \pm 50 \% \\
\text { Resist elasticity: } 60 \pm 5 \% \\
\text { Adhesive strength with main body: } \geq 50 \mathrm{Kgf} / \mathrm{cm}^{2}\end{array}$} \\
\hline Outside gasket & \multicolumn{3}{|c|}{$\begin{array}{c}\text { Hardness: } 35 \pm 5 \text { shore A } \\
\text { Tensile strength: } 80 \pm 30 \mathrm{Kgf} / \mathrm{cm}^{2} \\
\text { Elongation: } 300 \pm 50 \%\end{array}$} \\
\hline
\end{tabular}

Remarks: The main body and the inside gaskets were inserted into the resin via a hot-pressing method or a molding method, to prevent them from moving under a pressure of $2 \mathrm{Kgf}$ (at a depth of $20 \mathrm{~m}$ ). Combined tensile strength: $\geq 55 \mathrm{kgf} / \mathrm{cm}^{2}$.

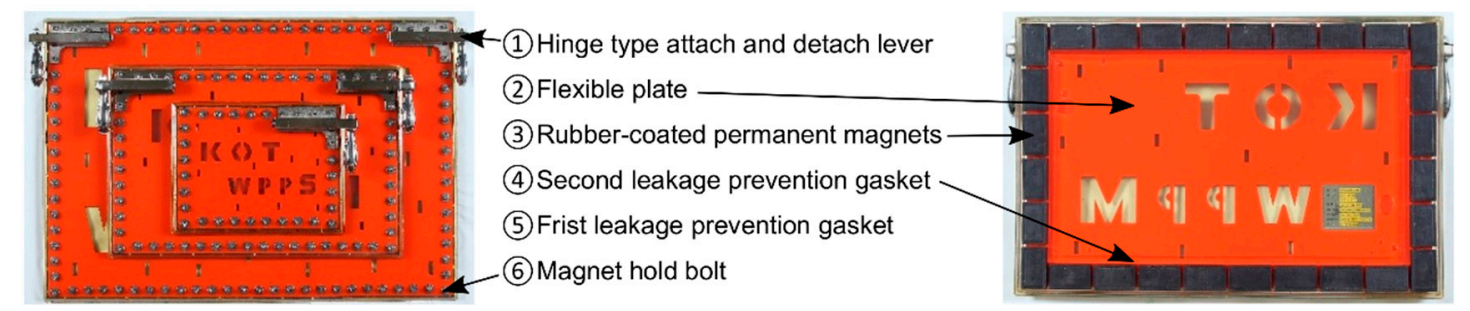

Figure 2. Magnetic-type liquid stop device (2017) [38].

\subsection{Types of Tests}

Since no standard guidelines are available for testing magnetic liquid stop devices, the following tests were performed to resolve the identified issues, such as practical ability to attach to flat and round surfaces, usability during sailing, and additional issues for the safe use of devices, with a focus on practical usability: water pressure test, attachment test, magnetic field test, and spark test. Attachment tests were performed when the ship was stationary, when the ship was sailing with the device attached to it, and on a round surface. Since the purpose of these tests was to verify the applicability and effectiveness of the device, this study did not consider the material properties, weight, and water temperature for every magnetic liquid stop device. The specific objectives, methods, and materials of each test were as follows.

(1) Water pressure and leak tests: These tests were conducted to verify the maximum depth at which the device can stop the inflow of seawater during an emergency. The test was only partly guided by the pressure vessel test guidelines of ASME Section VIII Division 1, because this was not a pressure vessel test [39]. The test apparatus was fabricated as shown in Figure 3. The diameter and height of the pressure vessel are both $1000 \mathrm{~mm}$, and the diameter of the steel plate is $1200 \mathrm{~mm}$. 
The magnetic liquid stop device was attached to the pressure vessel steel plate of the water pressure and leak test equipment, as shown in Figure 4. Once the steel plate was assembled, the pressure vessel was rotated by $180^{\circ}$ to simulate the condition of attachment to the hull bottom. Then, the vessel was filled with fresh water and pressurized with air. The experimental pressure was increased step-by-step to $0.15,0.5,0.75$, and $1.0 \mathrm{~kg} / \mathrm{cm}^{2}$. The amount of leaked water was measured for $1 \mathrm{~min}$ at each pressure step. In this test, only the WPP-M was used, considering the sizes and configuration of the holes in the leak test equipment. The test equipment included two steel plates: one with an $80 \mathrm{~mm}$ diameter hole, four $40 \mathrm{~mm}$ diameter holes, and four $20 \mathrm{~mm}$ diameter holes, and another with a $120 \mathrm{~mm}$ diameter hole. Since this was a general test for the usable pressure of the device, it was performed without considering the effects of various external forces such as weather and waves acting on an actual ship. Therefore, the test for the usable pressure of the device was performed in a static situation. In addition, because this test was performed in a state where the puncture is flat, the applicability of the device to uneven surfaces is unconfirmed.
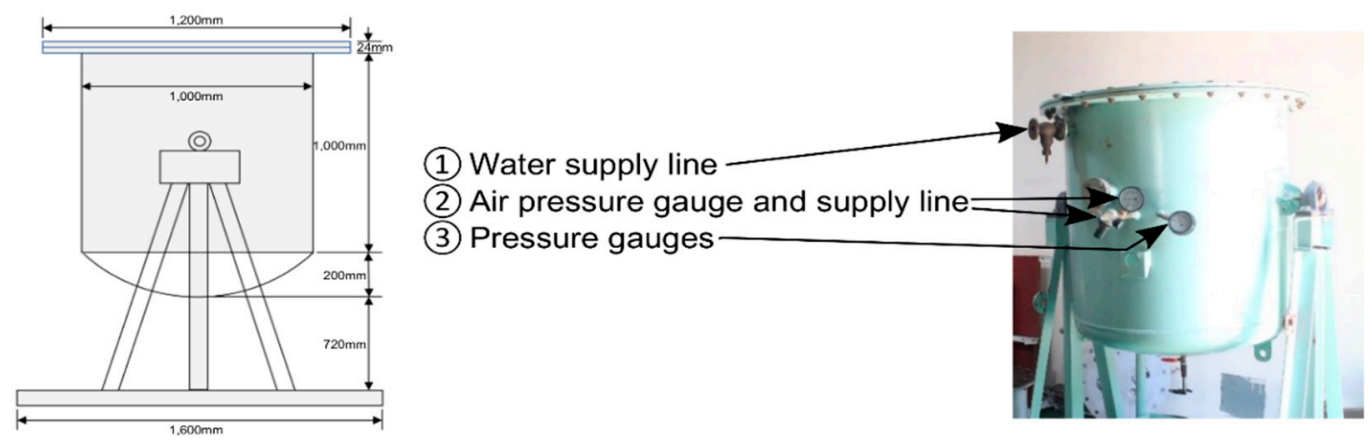

Figure 3. Water pressure test equipment [36].

(a)

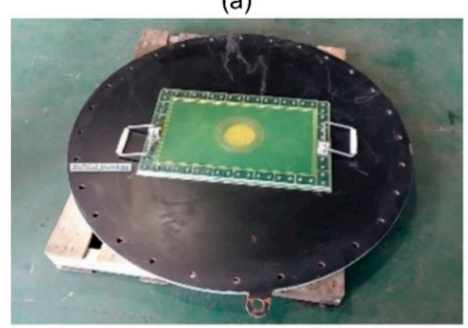

(d)

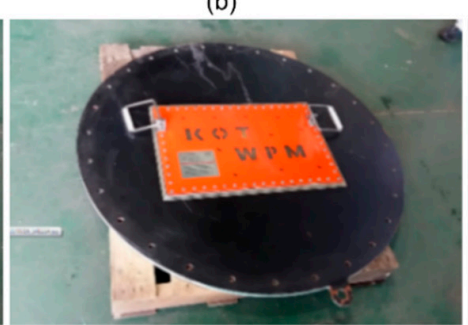

(c)

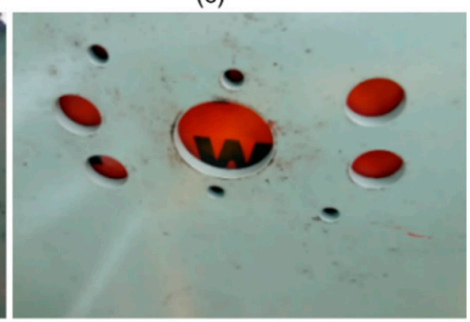

(e)

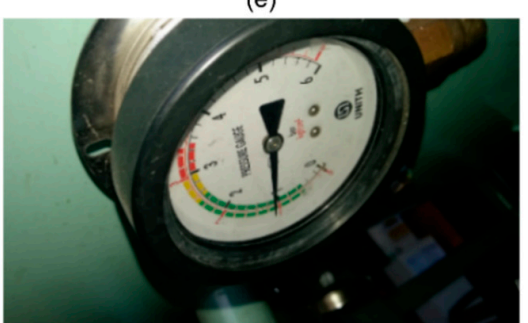

Figure 4. Water pressure tests (maximum pressure: $1.0 \mathrm{~kg} / \mathrm{cm}^{2}$ ). Clockwise from top left: (a) $120 \mathrm{~mm}$ hole steel plate; (b) attached condition of steel plate with $80 \mathrm{~mm}, 40 \mathrm{~mm}$, and $20 \mathrm{~mm}$ diameter hole, (c) opposite side teel plate $80 \mathrm{~mm}, 40 \mathrm{~mm}$, and $20 \mathrm{~mm}$ diameter hole; (d) test equipment assembly; (e) application of maximum pressure.

(2) Attachment tests: Three attachment tests were performed to verify the ability of the device to attach itself. First, to verify that the fabricated product attaches itself in the first place, an experiment was conducted to attach it to an actual ship. Second, to verify the state of the attachment during sailing, three magnetic liquid stop devices of different sizes were attached to the starboard side of the test ship. In most cases when this device is used, it is most likely that the 
ship is stationary owing to incidents such as collision or grounding, etc. However, considering a situation in which the device must be attached and the ship must be able to sail to a safe area, a test for the attachment during sailing was performed. The state of the attachment was checked in the presence of DNV-GL surveyors after each sailing trip. Third, the magnetic liquid stop devices were evaluated for proper attachment to round surfaces. To verify the attachment of the device to a round surface, the device was physically applied to a round surface. For the first test (Figure 5), the magnetic liquid stop device was applied to the berthed Korea Coast Guard Ship 1501, which was built in 2002. The ship has a displacement of 1500 tons, overall length (LoA) of $97.75 \mathrm{~m}$, and breadth of $14.0 \mathrm{~m}$. For this test, as shown in Figure 6, large, medium, and small size magnetic liquid stop devices were attached to the starboard side of the berthed ship at a depth of $2 \mathrm{~m}$, and a medium size magnetic liquid stop device was attached to the bottom at a depth of $3.6 \mathrm{~m}$ underwater and $3 \mathrm{~m}$ from the center of the hull. Figure 6 shows the attachment of the device to the starboard and bottom of the ship during the different stages of preparing the device: being on the boat outside the ship, diving in with the device, and attaching the device to the area as planned, according to the size of the devices. The test was conducted under the berthed condition of the vessel and without pressure from any puncture. When an actual puncture occurs, the surface of that area will be uneven in most cases. Therefore, this study has the limitation that the test is performed only on a flat surface.

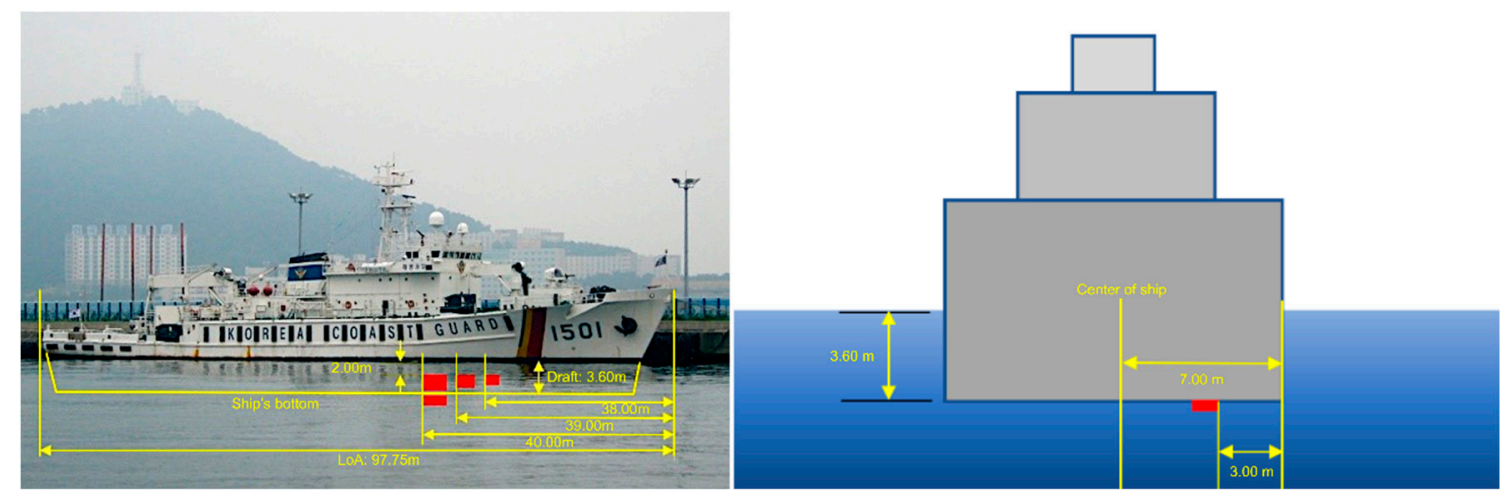

Figure 5. Conceptual representation of ship locations for the emergency magnetic liquid stop device attachment tests.

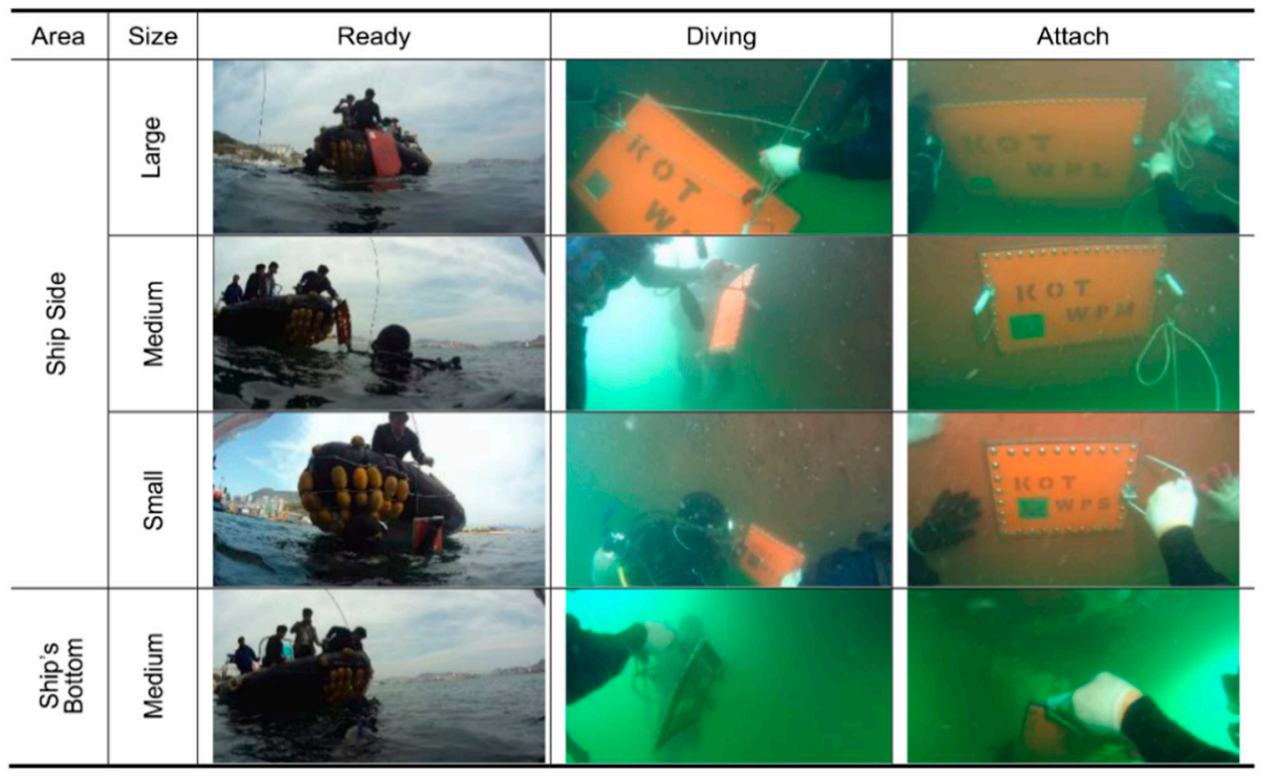

Figure 6. Attaching emergency magnetic liquid spill stoppers to the ship for attachment tests [36]. 
To test how effectively the magnetic liquid stop device can remain attached to the ship during sailing, an attachment status test during sailing was conducted on the 50-ton P-30 ship of the Busan Coast Guard. The P-30 is a patrol boat with a length of $28.7 \mathrm{~m}$, width of $5.4 \mathrm{~m}$, and maximum ship speed of $30 \mathrm{kts}$. Devices with three different sizes (WPP-L, WPP-M, WPP-S) were attached to the ship's starboard side. Then, the ship sailed for $5 \mathrm{~min}$ at 5 (772 m), 10 (1543 m), $12(1852 \mathrm{~m}), 15(2315 \mathrm{~m})$, and $18(2778 \mathrm{~m}) \mathrm{kts}$, and the attachment of the device was checked. The length of the test was based on the stopping distance of the ship at the maximum speed of $18 \mathrm{kts}$, which was $2550 \mathrm{~m}$. Therefore, a test length of 5 min could accommodate the time and distance needed for the stop when sailing at speeds between 5 and $18 \mathrm{kts}$. The water flow concept when the magnetic liquid stop devices are attached to a moving ship is shown in Figure 7. It shows a cross-section with the device attached to the ship's hull and the ship is sailing toward the right side. Seawater applies pressure to the device from the right to the left side during sailing. To this end, the drag force $\left(F_{D}\right)$ of the area subjected to water pressure during sailing was calculated using Equation (1) and compared with the holding force of the magnetic force. In Equation (1) $F_{D}[\mathrm{~N}]$, is the drag force, $C_{D}$ is the cube drag coefficient (1.05) [40], $A_{D}\left[\mathrm{~m}^{2}\right]$ is the projected area, $\rho\left[1,250 \mathrm{~kg} / \mathrm{m}^{3}\right]$ is the density, and $v[\mathrm{~m} / \mathrm{s}]$ is the ship speed. The block magnetic flux density (or B field) can be calculated using Equation (2) [41]. The terms and values for Equation (2) are presented in Figure 8. The magnetic fluxes according to the magnet shape and size were calculated using a magnetic flux calculator [42]. It is clarified in advance that the test was performed at the specific speed of the ship and did not consider various external forces such as wind speed and waves. In addition, the largest drag force during the test occurred when the devices were completely submerged; however, the fact that the devices were submerged when attached to the ship during the test in not always considered. Therefore, this study performs the test under the consideration that the biggest drag force occurs when the devices are completely submerged.

$$
\begin{gathered}
F_{D}=C_{D} \times \frac{1}{2} \rho v^{2} \times A_{D} \\
B=\frac{B_{r}}{\pi} \cdot\left(\arctan \left(\frac{L \cdot W}{\sqrt[2 z]{4 z^{2}+L^{2}+W^{2}}}\right)-\arctan \left(\frac{L \cdot W}{2(D+z) \sqrt{4(D+z)^{2}+L^{2}+W^{2}}}\right)\right)
\end{gathered}
$$

$B$ : Magnetic flux density ( $B$ field or magnetic induction)

$B_{r}$ : Remanence field, independent of the magnet's geometry

$\mathrm{Z}$ : Distance from a pole face on the symmetry axis $(\mathrm{mm})$

$L$ : Length of the block (mm)

$\mathrm{W}$ : Width of the block (mm)

$D$ : Thickness (or height) of the block (mm)

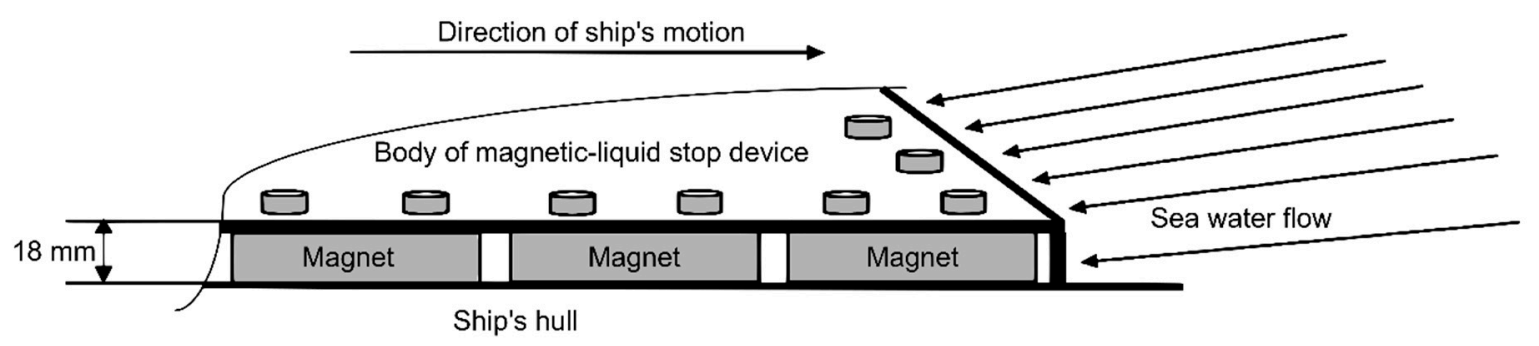

Figure 7. Water flow concept when a device is attached to the ship's hull. 


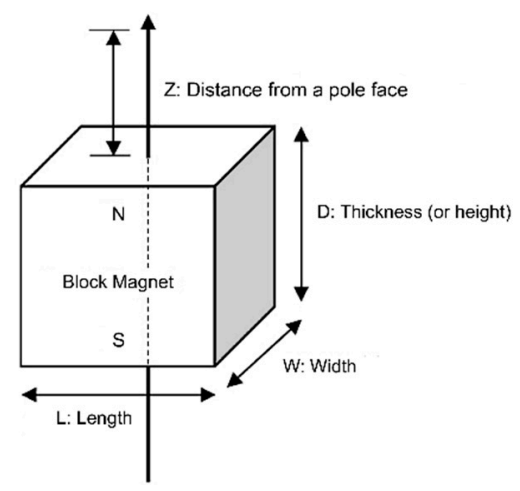

Figure 8. Size definition of the magnet.

The unit of length can be selected arbitrarily, provided that it is the same for all lengths. With respect to the attachable bend, the spacing between the magnets installed in the magnetic liquid stop device is $5 \mathrm{~mm}$, and their height is $10 \mathrm{~mm}$. The maximum allowable angle of the round surface can be calculated as shown in Figure 9 and Equation (3). In Equation (3), $2 \beta$ becomes $151.04^{\circ}$, indicating that the central angle $\theta$, which is calculated through as $\mathrm{L} / \mathrm{r}$ ( $\mathrm{L}$ is arc length, $\mathrm{r}$ is radius), of the round surface must be smaller than $151.04^{\circ}$. This angle is a limitation in the physical use of this device. Moreover, because the surface of the punctured hole will be uneven when an accidental puncture occurs, it is stated in advance that the device applied in the experiment has limitations in this regard, as the test is performed on a flat surface.

$$
2 \beta=\left(90^{\circ}-\operatorname{Sin} \alpha\right) \times 2
$$

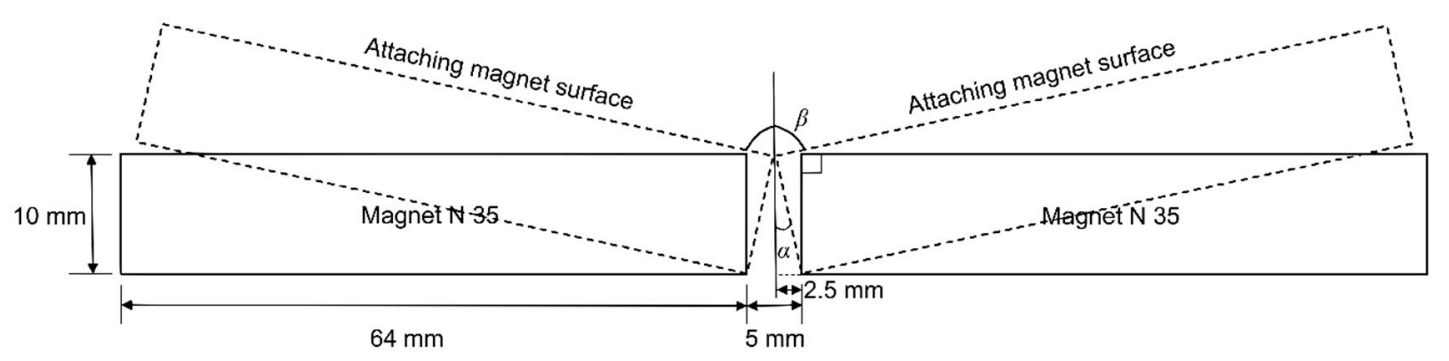

Figure 9. Attachable angle of magnetic liquid stop device.

(3) Magnetic field tests: A magnetic field strength above a certain limit can interfere with nearby wireless or radio communication or with the human body, affecting the electronic devices aboard a vessel $[43,44]$. The effect of the magnetic field on the ship's navigation system was examined during the storage of the magnetic liquid stop device and its attachment to the ship.

(a) Magnetic field tests in the berth

Figure 10 shows the MAGNA MG-701 test equipment and the method used to measure the magnetic field [45]. As shown in Figure 10, two magnetic field tests were performed. The elastic rubber-coated magnet used in the magnetic liquid stop device was fixed to one side, and the magnetic field was measured at 100, 250, 500, and $750 \mathrm{~mm}$ from the fixed position. Then, the magnetic liquid stop device was attached to a steel plate and the magnetic field strength at 250, 500, and $750 \mathrm{~mm}$ distances was measured. 

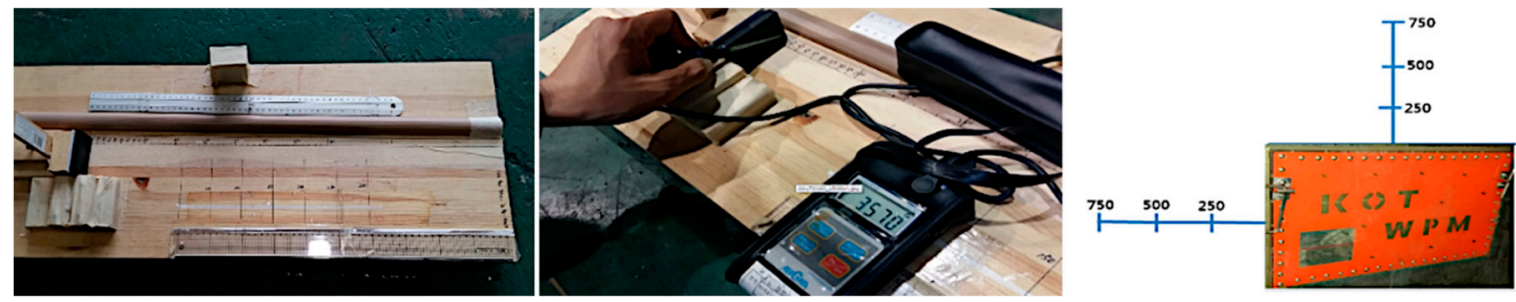

Figure 10. Magnetic field test. From left to right: fixing the magnet's position; checking its magnetic field; checking the magnetic field of the device.

(b) Magnetic field tests when sailing

To examine the possibility of a magnetic field affecting navigation and electronic equipment when the magnetic liquid stop device is used during sailing, the device was attached to the side of a 75-ton ship of the Busan Coast Guard, and the magnetic field was measured using a magnetic field meter (MAGNA MG-701). Three magnetic liquid stop devices were attached to the starboard side (WPP-M: 3 sets) and three were attached to the port side (WPP-M: 3 sets). The measurements were performed at a ship speed of $18.4 \mathrm{kts}$ off the coast of Busan Port (North: $35^{\circ} 06^{\prime}$ N. East: $120^{\circ} 08^{\prime}$ E). Four locations on the ship were selected for measuring the magnetic fields: forecastle, poop, midship, and positions of attachment of the device.

(4) Spark tests: Sparks generated during the installation of the magnetic liquid stop device can be dangerous if there are flammables in the vicinity [46]. Therefore, an experiment was conducted to check the generation of sparks by the magnetic liquid stop device. The spark test was conducted to determine the occurrence of static electricity when the magnetic liquid stop device is attached to a ship. For this test, a 10-mm-thick magnet was free-dropped from a height of $500 \mathrm{~mm}$ (Figure 11) and observed for the occurrence of static electricity. The dimensions of the test equipment for the free-drop test were $750 \mathrm{~mm}$ (length) $\times 500 \mathrm{~mm}$ (height) $\times 100 \mathrm{~mm}$ (width), as shown in Figure 11. A steel plate (diameter: $200 \mathrm{~mm}$ ) was placed on the test equipment and a rubber-coated magnet [39 mm (length) $\times 64 \mathrm{~mm}$ (width) $\times 10 \mathrm{~mm}$ (thickness)] was freely dropped. The test was conducted in a dark environment to check for the occurrence of sparks, and the occurrence of static electricity was checked after five free drops. This test is limited to the confirmation of whether sparks are generated upon contact with the ship's hull or steel plate when using the device.

(a)

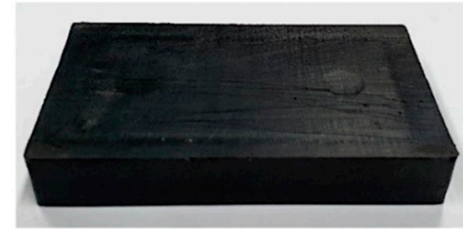

(c)

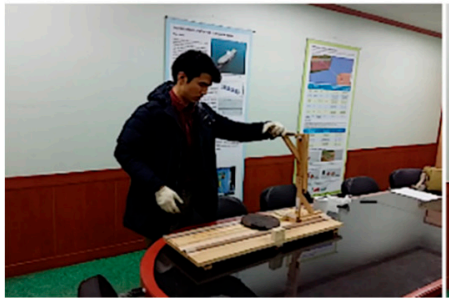

(b)

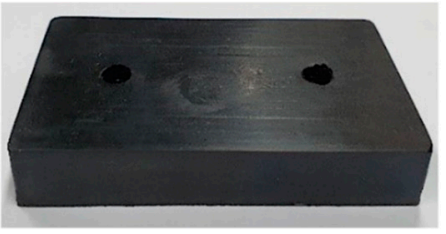

(d)

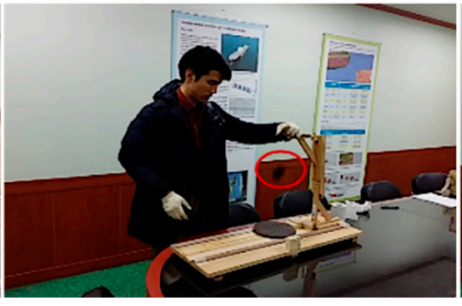

(e)

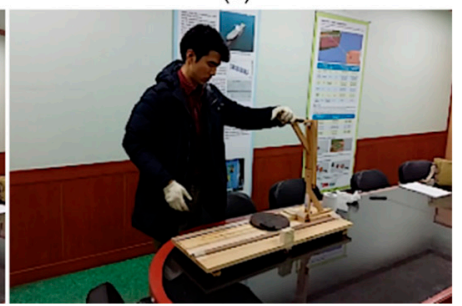

Figure 11. Top panel: Rubber-coated magnet for magnetic liquid stop device (a) front on the left and (b) back on the right). Bottom panel: Magnet free-drop spark test procedure: (c) stand by; (d) drop; and (e) confirm. 


\subsection{Cost-Benefit Analysis}

Even if the magnetic liquid stop device is shown to be effective, a cost-benefit analysis still needs be undertaken before it is feasible to install the device in a ship. To that end, a cost-benefit analysis was performed in accordance with the FSA guideline of the IMO [47]. In this evaluation, the gross Cost of Averting a Tonne of Spilled oil (CATS) was used as shown in Equation (4), as recommended in the guideline. In terms of the guideline, when the effects of the proposed RCOs are only applied to an oil spill, there is a cost-benefit when $\Delta C<\Delta S C$, where $\Delta C$ is the expected cost of the RCO during the lifetime of the vessel, and $\triangle S C$ is the expected benefit of the RCO [48]. The cost-benefit of the RCO is distributed over the lifetime of the ship and may require financing every year or at certain intervals. The lifetime of a ship was assumed to be 25 years, according to the guideline. The net present value (NPV) of the RCO was calculated using Equation (5) [18]. According to the FSA guideline, $S C(V)$ can be calculated through Equation (6) [47]. However, the actual $F_{\text {Assurance }}$ and $F_{\text {Uncertainty }}$ are difficult to calculate in this state because of uncertainty. Therefore, this study applied the F factor used in the previous study [49].

$$
\begin{gathered}
\text { CATS }=\frac{\Delta C}{\Delta R E} \\
N P V=A+\frac{X}{(1+r)}+\frac{X}{(1+r) 2}+\frac{X}{(1+r) 3}+\cdots+\frac{X}{(1+r) T}=A+\sum_{t=1}^{T} \frac{X}{(1+r)^{t}} \\
S C(V)=F_{\text {assurance }} \times F_{\text {Uncertainty }} \times f(V)
\end{gathered}
$$

$X=$ Cost or benefit of RCO for any given year

$A=$ Amount spent initially for the implementation of RCO

$r=$ Discount rate

$T=25$ (years)

$\Delta C=$ Expected cost of the RCO during the lifetime of the vessel [18]

$\Delta R_{E}=$ Risk reduction per ship, in terms of tonnes of oil spilled prevented $\left(\Delta \mathrm{R}_{\mathrm{E}}\right)$, implied by the $\mathrm{RCO}$ during the lifetime of the vessel [18]

$\triangle S C=($ Expected SC without the RCO $)-($ Expected SC with the RCO $)=$ Expected benefit of the RCO [47]

$F_{\text {Assurance }}=$ Allowing for society's willingness to pay to avert accidents

$F_{\text {Uncertainty }}=$ Allowing for uncertainties in the cost information related to oil spill accidents

$f(V)=$ Cost per unit oil spilled decreases with the spill size in US\$ per tonne oil spilled

$\mathrm{F}$ is an "assurance parameter", reflecting the fact that spending resources on preventing oil spills is always preferable to accepting similar costs related to an actual spill. According to Vanem et al. (2007), if an F factor of 1.5 is applied, the restoring cost for 1 tonne of oil spilled is approximately USD 60,000 [18]. Thus, the criterion of Equation (7) was applied to the cost-benefit assessment for the magnetic liquid stop device in this study. F might have a value above 1, as determined by the decision makers. An exact value for the assurance factor $\mathrm{F}$ was not established in this study because of the uncertainty of environmental costs [18]. The exact $\mathrm{F}$ factor has not been determined yet. The previous study recommended an $\mathrm{F}$ factor of 1.5 based on the uncertainty in environmental costs. Evidently, a smaller F factor indicates higher cost-benefit. In this study, an F factor of 1.5 was considered, as the range from 1.0 to 1.5 suggested in the previous study was believed to be acceptable.

$$
\text { Cost of averting a spill }<\mathrm{F} \times \text { Cost of an occurred spill }
$$

CATS $<$ F $\times$ USD 40,000 (Global average clean-up cost USD 16,000 + Global average estimate for the cost of environmental damages USD 24,000)

CATS $<$ USD 60,000 (applied F factor 1.5 for the global criterion) [18]. 


\section{Results}

\subsection{Water Pressure and Leak Test}

The leakage of water at different pressures in the water pressure test is outlined in Table 2. The test results show that $0.7 \mathrm{~L}$ of water leaked for $1 \mathrm{~min}$ at $1.0 \mathrm{~kg} / \mathrm{cm}^{2}$, but no water leaked at other pressures. At sea, the water pressure at a depth of $7.3 \mathrm{~m}$ is approximately $0.75 \mathrm{~kg}$, according to the equation in the remark in Table 2. That is, through the water pressure test, the device can be applied from seawater to ships with about 7.3 draft. Although water began leaking at a pressure of $1.00 \mathrm{~kg} / \mathrm{cm}^{2}$, the device is still reasonably applicable at a depth of $9.8 \mathrm{~m}$, in terms of its ability to reduce the amount of outflow even if it is not completely blocked.

Table 2. Results of water pressure tests.

\begin{tabular}{|c|c|c|c|c|c|c|}
\hline \multirow{2}{*}{ No } & \multirow{2}{*}{ Model } & \multicolumn{5}{|c|}{ Pressure } \\
\hline & & $0 \mathrm{kgf} / \mathrm{cm}^{2}$ & $0.25 \mathrm{kgf} / \mathrm{cm}^{2}$ & $0.5 \mathrm{kgf} / \mathrm{cm}^{2}$ & $0.75 \mathrm{kgf} / \mathrm{cm}^{2}$ & $1.0 \mathrm{kgf} / \mathrm{cm}^{2}$ \\
\hline \multirow{2}{*}{1} & WPP-M & No leakage & No leakage & No leakage & No leakage & Leakage \\
\hline & Leak $Q^{\prime}$ ty & - & - & - & - & $0.7 \mathrm{~L} / 1 \mathrm{~min}$ \\
\hline
\end{tabular}

Remark: $\mathrm{P}=\rho \mathrm{gz}=1025 \frac{\mathrm{kg}}{\mathrm{m}^{3}} \times 9.8 \mathrm{~m} / \mathrm{s}^{2} \times 7.3 \mathrm{~m}=73,328.5 \mathrm{~kg} / \mathrm{m} \cdot \mathrm{s}^{2} \cdot \mathrm{P}=73,328.5 \mathrm{~kg} / \mathrm{m} \cdot \mathrm{s}^{2}=0.748 \mathrm{kgf} / \mathrm{cm}^{2}$.

\subsection{Attachment Test}

\subsubsection{Flat Surface Attachment Test in Berth}

The attachment tests on the berthed ship confirmed that all the devices attached effectively. The time required, after the diver received the device, attaching it to the ship's bottom and to the ship's side took $82 \mathrm{~s}$ (longest attachment time) and $38 \mathrm{~s}$ (shortest attachment time), respectively. This indicates that it is possible to attach the device to the side and bottom of the ship in a relatively short time. Since this time depends on many factors such as the weather at sea and the diver's gender and age, we can attest that the device can be installed in a relatively short time.

\subsubsection{Attachment Test during Sailing}

Table 3 shows the results of the drag force $\left(F_{D}\right)$ of the area subjected to water pressure during sailing, which was calculated using Equation (1), and Table 4 shows the block magnet flux density, which was calculated using Equation (2).

Table 3. Results of $\mathrm{F}_{\mathrm{D}}$ calculation.

\begin{tabular}{|c|c|c|c|}
\hline $\begin{array}{c}\text { Size } \\
\text { (Length and Breadth) }\end{array}$ & $\begin{array}{c}\text { Large }(\text { WPP-L) } \\
(0.638 \mathrm{~m} \times 0.018 \mathrm{~m}) \\
\left(\mathrm{A}_{\mathrm{D}}=0.011484 \mathrm{~m}^{2}\right)\end{array}$ & $\begin{array}{l}\text { Medium }(\text { WPP-M) } \\
(0.431 \mathrm{~m} \times 0.018 \mathrm{~m}) \\
\left(\mathrm{A}_{\mathrm{D}}=0.007758 \mathrm{~m}^{2}\right)\end{array}$ & $\begin{array}{c}\text { Small (WPP-S) } \\
(0.293 \mathrm{~m} \times 0.018 \mathrm{~m}) \\
\left(A_{D}=0.005274 \mathrm{~m}^{2}\right)\end{array}$ \\
\hline Ship Speed & $F_{D} N$ & $F_{D} N$ & $F_{D} N$ \\
\hline (1) $5 \mathrm{kts}(2.572 \mathrm{~m} / \mathrm{s})$ & 40.8807 & 27.6169 & 18.7744 \\
\hline (2) $10 \mathrm{kts}(5.144 \mathrm{~m} / \mathrm{s})$ & 163.523 & 110.468 & 75.0975 \\
\hline (3) $12 \mathrm{kts}(6.1728 \mathrm{~m} / \mathrm{s})$ & 235.473 & 159.073 & 108.14 \\
\hline (4) $15 \mathrm{kts}(7.716 \mathrm{~m} / \mathrm{s})$ & 367.926 & 248.552 & 168.969 \\
\hline (5) $18 \mathrm{kts}(9.2592 \mathrm{~m} / \mathrm{s})$ & 529.814 & 357.915 & 243.316 \\
\hline
\end{tabular}

Remark: Beaufort wind scale: 5 (17-21 kts), Wave scale: 4 (moderate, $1.25-2.3 \mathrm{~m})$. 
Table 4. Magnetic holding force (N) [42].

\begin{tabular}{ccccccc}
\hline \multicolumn{7}{c}{ Type and Size of Magnet } \\
Size & Gauss (1 ea) & $\begin{array}{c}\text { Holding Force } \\
\text { per Magnet (Kgf) }\end{array}$ & $\begin{array}{c}\text { Holding } \\
\text { Force per } \\
\text { Magnet (N) }\end{array}$ & $\begin{array}{c}\text { Number of } \\
\text { Magnets }\end{array}$ & $\begin{array}{c}\text { Total } \\
\text { Holding } \\
\text { Force (Kgf) }\end{array}$ & $\begin{array}{c}\text { Total } \\
\text { Holding } \\
\text { Force(N) }\end{array}$ \\
\hline Large & 1759 & 7.13 & 69.88 & 42 ea & 299.46 & 2934.96 \\
Medium & 1759 & 7.13 & 69.88 & 28 ea & 199.64 & 1956.64 \\
Small & 1759 & 7.13 & 69.88 & 16 ea & 114.08 & 1118.08 \\
\hline
\end{tabular}

Remark: Distance from magnet surfaces: $0.1 \mathrm{~mm}$; Coating thickness of magnet: $0.1 \mathrm{~mm}$.

As shown in the results in Table 3, the maximum pressure that the attached large magnet was subjected to in relation to the ship speed was $529.814 \mathrm{~N}$. According to the results shown in Table 4, the holding force of the large magnet is $2934.96 \mathrm{~N}$. Thus, the holding force was shown by computation to be sufficiently larger than the drag force, confirming that the device can maintain its attached state. This was verified when the magnetic liquid stop device was applied to an actual ship, and it was found that the device maintained its attached state at the test speeds of the ship. Figure 12 shows photographs of the attachment status test during the sailing of the ship P-30. This experiment was focused to check whether the device maintained its attached state. However, it can be inferred from Figure 12 that there was almost no sideways movement of the devices when the attachment position before sailing and after sailing are compared with the letters on the side of the ship.
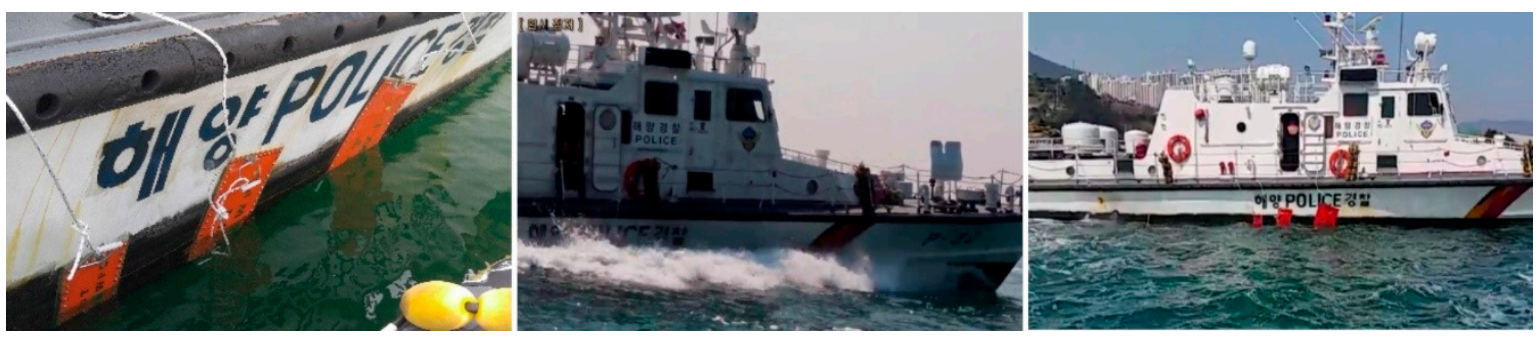

Figure 12. Emergency liquid spill stoppers during attachment tests when sailing. From the left to right: Before sailing, during sailing, after sailing.

\subsubsection{Attachment Test for Round Surfaces}

Table 5 shows the attachable angles of the magnetic liquid stop devices using Equation (3). Thus, it was found that the large and medium size devices are attachable to a round surface with the minimum diameters of 708 and $498 \mathrm{~mm}$, respectively; for the small size device, the diameter must be at least $290 \mathrm{~mm}$.

Table 5. Round surface attachable angle calculation.

\begin{tabular}{cccc}
\hline & Large (WPP-L) & Medium (WPP-M) & Small (WPP-S) \\
\hline Width $\times$ length $(\mathrm{mm})$ & $933 \times 638$ & $657 \times 431$ & $381 \times 293$ \\
Maximum angle of round surfaces & $151.04^{\circ}$ & $151.04^{\circ}$ & $151.04^{\circ}$ \\
$\quad 700 \mathrm{~mm}$ & $151.00^{\circ}$ & $106.34^{\circ}$ & $61.67^{\circ}$ \\
(external diameter: $708 \mathrm{~mm})$ & & & \\
$\quad \begin{array}{c}1000 \mathrm{~mm} \\
\text { (external diameter: } 1008 \mathrm{~mm})\end{array}$ & $106.07^{\circ}$ & $74.69^{\circ}$ & $43.31^{\circ}$ \\
Minimum required diameter to be less than 151.04 & $708 \mathrm{~mm}\left(151.00^{\circ}\right)$ & $498 \mathrm{~mm}\left(151.18^{\circ}\right)$ & $290 \mathrm{~mm}\left(150.55^{\circ}\right)$ \\
\hline
\end{tabular}

Figure 13 demonstrates the attachment of the magnetic liquid stop devices when they were physically applied to round surfaces with the external diameters of $708 \mathrm{~mm}$ (internal diameter: $700 \mathrm{~mm}$ ) and $1108 \mathrm{~mm}$ (internal diameter: $1100 \mathrm{~mm}$ ). The results showed that the devices of all three sizes attached normally. This test confirmed that the magnetic liquid stop device can be effectively applied to round surfaces as well because it is made of flexible rubber and polyurethane materials. 


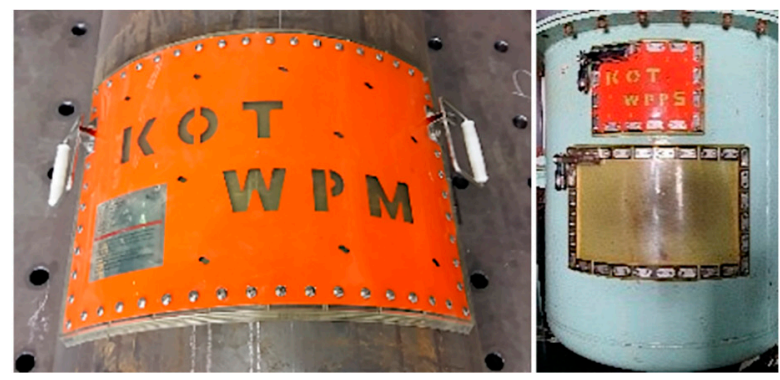

Figure 13. Round surface attachment test (left: $700 \mathrm{~mm}$ (pipe), right: $1000 \mathrm{~mm}$ (cylinder)).

\subsection{Magnetic Field Test}

\subsubsection{Magnetic Field Test in the Berth}

The magnetic field test was conducted to verify whether the magnetic force of the magnetic liquid stop device affects a ship's navigation and electronic equipment. Before measuring the device's magnetic field, the gauss calculation, according to the shape and size of the N-35 magnet, was applied to the gauss graph calculator [42]. As a result, the gauss values with respect to their distances could be obtained as shown in Table 6. The gauss graphs by distance were predicted as shown in Figure 14.

Table 6. Gauss calculation by distance [42].

\begin{tabular}{|c|c|c|c|c|c|c|c|c|c|}
\hline \multicolumn{10}{|c|}{$\begin{array}{l}\text { Type and Size of Magnet } \\
(\mathrm{Nd}-\mathrm{Fe}-\mathrm{B} \text { Magnet }(\mathrm{N}-35), \text { Length } \times \text { Width } \times \text { Height: } 53 \mathrm{~mm} \times 28 \mathrm{~mm} \times 6 \mathrm{~mm}\end{array}$} \\
\hline Distance (mm) & 50 & 100 & 200 & 300 & 400 & 500 & 600 & 700 & 750 \\
\hline Gauss (13 ea) & 109.24 & 31.08 & 8.47 & 3.79 & 2.04 & 1.22 & 0.78 & 0.52 & 0.43 \\
\hline Gauss (9 ea) & 110.47 & 31.85 & 8.46 & 3.53 & 1.77 & 1.00 & 0.61 & 0.4 & 0.33 \\
\hline
\end{tabular}

Remark: Distance from magnet surfaces: $0.1 \mathrm{~mm}$; Coating thickness of magnet: $0.1 \mathrm{~mm}$; Earth magnetic field strength: $0.25-0.65$ gauss.
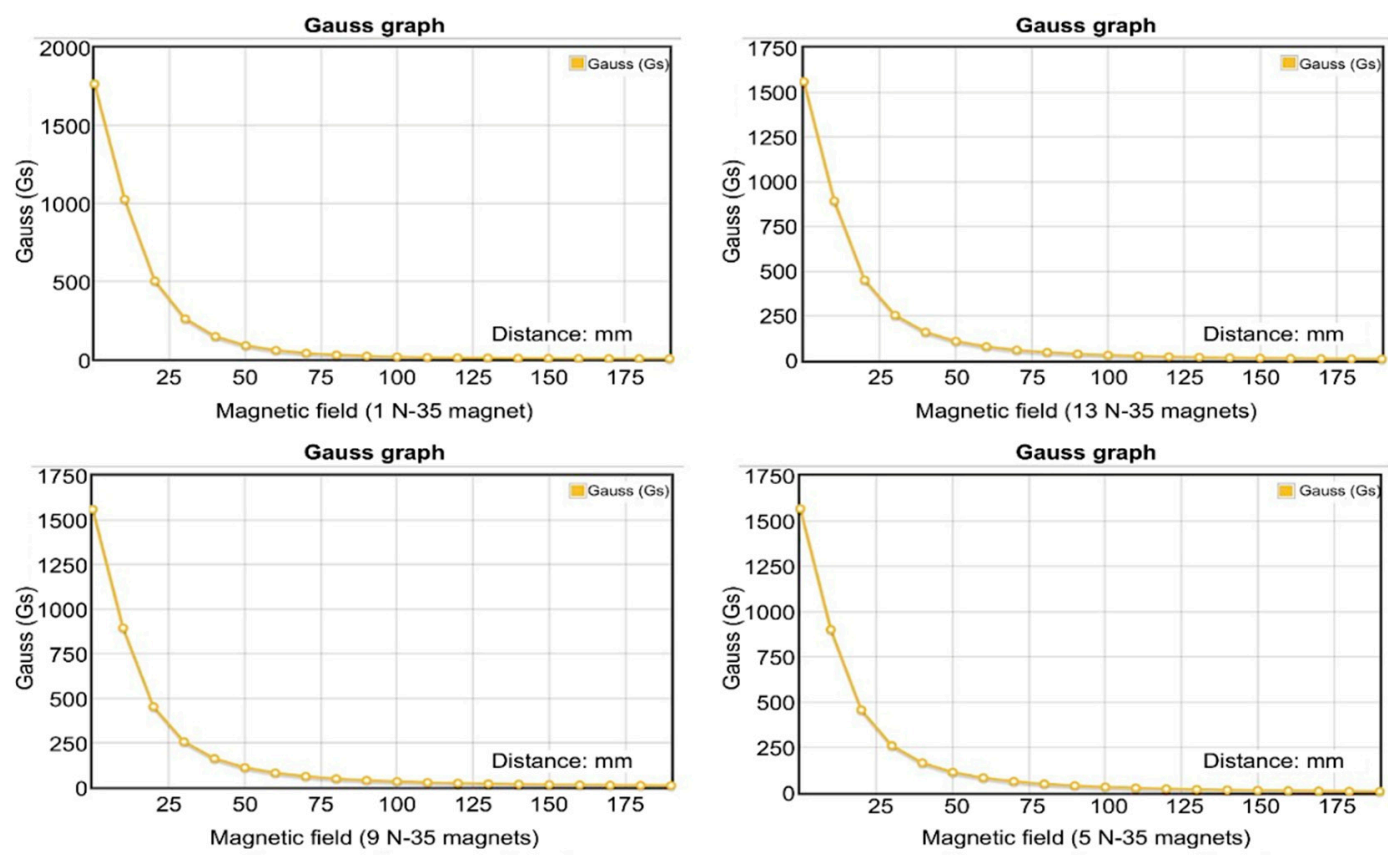

Figure 14. Gauss graph of N-35 magnets by distance [42]. 
The magnetic field of one N-35 magnet with the dimensions of $53 \mathrm{~mm}$ (length) $\times 28 \mathrm{~mm}$ (width) $\times 6 \mathrm{~mm}$ (height) becomes 2.02 Gauss at $200 \mathrm{~mm}$ and 0.61 Gauss at $300 \mathrm{~mm}$, as shown in the top left plot of Figure 14. For the large device, 13 magnets were installed on the longest side. When the total length of these magnets was $689 \mathrm{~mm}$, the magnetic field was 8.47 Gauss at $200 \mathrm{~mm}$ and 0.78 Gauss at $600 \mathrm{~mm}$, as shown in the top right plot of Figure 14. In the case of the medium device, when the total length of the magnets along the longest side was $477 \mathrm{~mm}$, the magnetic field was 8.46 Gauss at $200 \mathrm{~mm}$ and 0.61 gauss at $600 \mathrm{~mm}$, as shown in the bottom left plot of Figure 14. Finally, in the case of the small device, when the total length of the magnets along the longest side was $265 \mathrm{~mm}$, the magnetic field was 7.31 gauss at $200 \mathrm{~mm}$ and 0.64 gauss at $500 \mathrm{~mm}$, as shown in the bottom right plot of Figure 14. Therefore, the magnetic liquid stop devices have almost no magnetic field capable of affecting navigation and electronic equipment when located at distances at least $600 \mathrm{~mm}$ away from the equipment.

The test results showed that the strongest magnetic field of 3570 gauss was measured when one elastic rubber coated magnet was attached to the bottom. However, 1 gauss of magnetic field was measured at $250 \mathrm{~mm}$, and no magnetic field was measured at $750 \mathrm{~mm}$ or farther away, as shown in Table 7. When the device was attached to a steel plate surface, no magnetic field was measured farther away than $500 \mathrm{~mm}$, as shown in Table 7 . These results confirm that the magnetic liquid stop device does not affect electronic equipment at the distance of $750 \mathrm{~mm}$ or further, even if conservatively evaluated.

Table 7. Magnetic field measurement of elastic-rubber-coated magnet and magnetic liquid stop device.

\begin{tabular}{cccccc}
\hline \multirow{2}{*}{ No } & Direction & \multicolumn{4}{c}{ Measurement Result (Gauss) } \\
\cline { 3 - 6 } & & Contact & $\mathbf{2 5 0} \mathbf{~ m m}$ & $\mathbf{5 0 0} \mathbf{~} \mathbf{m}$ & $\mathbf{7 5 0} \mathbf{~ m m}$ \\
\hline 1 & Upper & 320 & 1 & 0 & 0 \\
2 & Down & 3570 & 1 & 1 & 0 \\
3 & Width & 500 & 1 & 0 & 0 \\
4 & Length & 140 & 1 & 0 & 0 \\
5 & Width/Length & 55 & 1 & 0 & 0 \\
6 & Left/Right & 180 & 1 & 0 & 0 \\
\hline
\end{tabular}

\subsubsection{Magnetic Field Tests During Sailing}

Magnetic field measurements from the four locations on the ship (attached, forecastle, poop, and midship) indicated that the maximum value of the magnetic field was 1 gauss. This suggests that there is almost no magnetic field in relation to the Earth's magnetic field. Thus, it was found that the device in the storage and attached positions does not affect the navigation and other electronic equipment aboard the ship.

The results shown in Table 8 confirmed that sparks arising from collisions did not occur owing to the paint applied to the hull and the elasticity of the rubber applied to the magnet. For this test, a 10-mm-thick magnet was free-dropped from a height of $500 \mathrm{~mm}$ and observed for the occurrence of static electricity. The test equipment dimensions were $750 \mathrm{~mm}$ (length) $\times 500 \mathrm{~mm}$ (height) $\times 100 \mathrm{~mm}$ (width). The equipment was made for free-drop test, as shown in Figure 11. A steel plate (diameter: $200 \mathrm{~mm}$ ) was placed on the test equipment, and a rubber-coated magnet [39 $\mathrm{mm}$ (length) $\times 64 \mathrm{~mm}$ (width) $\times 10 \mathrm{~mm}$ (thickness)] was freely dropped.

Table 8. Results of the free-drop spark test.

\begin{tabular}{ccccccc}
\hline \multirow{2}{*}{ Model } & \multicolumn{6}{c}{ Measurement Result (X: Non Spark, O: Spark) } \\
\cline { 3 - 7 } & KOT MAG. 50 & $\mathbf{1}$ & $\mathbf{2}$ & $\mathbf{3}$ & $\mathbf{4}$ & $\mathbf{5}$ \\
\hline 1 & K & $\mathrm{X}$ & $\mathrm{X}$ & $\mathrm{X}$ & $\mathrm{X}$ \\
\hline
\end{tabular}




\subsection{Cost-Benefit Analysis for Magnetic Liquid Stop Device as a Risk Control Option}

The cost-benefit analysis applied according to the IMO FSA was based on the cost of the equipment used in the above tests. The costs of the magnetic liquid stop devices used in the tests were estimated as shown in Tables 9 and 10, considering the international costs of materials and the business profits. The costs of the parts used in the equipment at the time of manufacturing in South Korea were checked. Although the material and labor costs vary with country, it was assumed that the material costs did not change significantly among countries according to international prices. In the case of WPP-L, the cost is USD 6000 (KOT), but using the same product over 25 years will carry uncertainty for maintaining the magnetism of the rubber magnet. Thus, considering the interannual variation of the magnetic liquid stop device (10 years, $1.7 \%$ ), it was assumed that the service life is 10 years and 2.5 sets were kept for 25 years over the ship's lifetime. Therefore, a factor of 2.5 times the price of each size of the device was applied. For the labor cost, $67 \%$ of the labor cost was added considering that as of 2018 , the country with the highest minimum wage is Luxembourg at USD 28,603.9, and it is USD 17,159.7 in South Korea. Under these assumptions, the costs of the devices are outlined in Table 9. According to this, the WPP-L costs USD 15,000 for 25 years. Based on this, $\triangle C$ and CATS were determined using Equations (4) and (5), respectively. $\triangle \mathrm{C}(\mathrm{NPV})$ was calculated using Equation (5), and the CATS was calculated using Equation (4). For this calculation, the life span of the ship was considered to be 25 years according to the FSA guideline, and depreciation of 5\% per year was applied [47].

Table 9. Costs of equipment.

\begin{tabular}{ccc}
\hline Size $(\mathbf{m m})$ & Price (USD) & Total Cost for 25 Years (USD) \\
\hline Large $(933 \times 638)$ & $6000 \times 2.5^{*}=15,000$ & 15,000 \\
Middle $(657 \times 431)$ & $3000 \times 2.5=7500$ & 7500 \\
Small $(381 \times 293)$ & $2000 \times 2.5=5000$ & 5000 \\
\hline
\end{tabular}

* The usable age of one piece of magnetic stopper is 10 years. Therefore, it is assumed that 2.5 magnetic stoppers are needed in a 25-year lifetime.

Table 10. Cost of Averting a Tonne of Spilled oil (CATS) by size of magnetic liquid stop device.

\begin{tabular}{cccc}
\hline & $\begin{array}{c}\text { Risk Reduction, } \Delta R E \\
\text { Oil Spill Reduction }\end{array}$ & Cost, $\Delta C$ & CATS $=\frac{\Delta C}{\Delta R E}$ \\
\hline Tonnes of Oil Spill Prevented & USD & USD \\
\hline Large & 0.33 & 19,228 & 58,267 \\
(WPP-L) & 1 & 19,228 & 19,228 \\
& 10 & 19,228 & 1922.8 \\
& 100 & 19,228 & 192.28 \\
\hline Medium & 0.18 & 10,319 & 57,328 \\
(WPP-M) & 1 & 10,319 & 10,319 \\
& 10 & 10,319 & 1031.9 \\
& 100 & 10,319 & 103.19 \\
\hline Small & 0.13 & 7819 & 60,146 \\
(WPP-S) & 1 & 7819 & 7819 \\
& 10 & 7819 & 781.9 \\
\hline
\end{tabular}

(1) Per ship lifetime, assumed to be 25 years

(2) Includes net present value (NPV) at $5 \%$ per year where relevant

(3) Reduced Potential Loss of Cargo (PLC) only.

The CATS for the large size device is USD 19,228 under the assumption that 1 tonne of oil spill is prevented in the event of oil spill caused by hull damage, regardless of the ship size. The calculation results can be found in Table 10 . 
Certainly, the CATS can be decreased if further oil spills can be prevented. Vanem et al. (2008) claimed that cost effectiveness is assured if CATS $<$ USD 60,000 when an F factor of 1.5 is applied. Thus, cost effectiveness can be confirmed if at least 0.33 tonnes of oil spill can be prevented by the magnetic liquid stop device used in this experiment [49]. Similarly, it can be considered that the device, as an IMO FSA RCO, is cost effective if approximately 0.18 tonnes of oil spill for the medium-size device and approximately 0.13 tonnes of oil spill for the small-size device can be prevented. The cost effectiveness is more certain if we consider that the crude oil price per barrel was around USD 100 at the time when Vanem et al. conducted their study (December 2007), and it was around USD 50 in February 2020 [50].

\section{Discussion}

In this study, an advanced magnetic liquid stop device was developed, and its effects were experimentally verified by applying it to actual ships and tests. Furthermore, to verify the practical applicability of the developed device, a cost-benefit analysis was performed according to the IMO's FSA guideline. It was concluded that the developed device was sufficiently applicable as an RCO. As identified from previous studies, there are some limitations associated with the practical usability of magnetic liquid stop devices in terms of resistance to pressure, efficient attachment of the devices, interference from their magnetic fields, and the potential to generate sparks. This study investigated an improved magnetic-type waterproof oil spill stop devices for ships, and it was proven that the device could partly address the disadvantages of previously developed devices. To prove this, this study carried out tests for water pressure and leakage, attachment (on a berthed vessel, sailing vessel, and round surfaces), magnetic fields, and spark generation. Nevertheless, this study has some limitations as discussed below. In addition, it was clarified that this study mainly focused on the applicability of the equipment, and the tests were conducted accordingly.

In the water pressure and leak test, there was no leakage up to a pressure of $0.75 \mathrm{~kg} / \mathrm{cm}^{2}$, and approximately $0.7 \mathrm{~L}$ of water leaked at a pressure of $1.0 \mathrm{~kg} / \mathrm{cm}^{2}$. This confirmed that oil leaks from a ship's hull can be stabilized down to the depth of $9.8 \mathrm{~m}$ when the standard density of seawater of $1025 \mathrm{~kg} / \mathrm{m}^{3}$ is applied. In this study, the usability of the device was verified at the conditions in which a pressure of $1.0 \mathrm{~kg} / \mathrm{m}^{2}$ can occur, such as when a puncture occurs at $9.8 \mathrm{~m}$ under the sea. In the water pressure and leak test, the draft for ships limits the depth to $9.8 \mathrm{~m}$ or less, and the maximum pressure is set at $1.0 \mathrm{~kg} / \mathrm{cm}^{2}$. This means that it cannot be universally applied to all ships, particularly to deep draft vessels. In addition, the water-pressure and leak tests were performed under stationary conditions; the results can differ for a moving ship and for a marine environment with a different water temperature and salinity. Moreover, in this study, the actual puncture of a ship was not considered, which might have pressure caused by outflow or inflow liquid. Therefore, further research needs to be conducted to overcome this limitation.

The device remained attached during sailing, even at a speed of $18.2 \mathrm{kts}$. A test duration of $5 \mathrm{~min}$ was used in consideration of the stopping distance required when the engine was stopped at each speed. According to Tables 3 and 4, the drag force, $\mathrm{F}_{\mathrm{D}}$, of WPP-L was $529.814 \mathrm{~N}$ at $18 \mathrm{kts}$, and the magnetic holding force was $2934.96 \mathrm{~N}$. Since the holding force was approximately 5.5 times stronger than the drag force, it is considered that the device can sufficiently endure general sea weather conditions. However, the result of this test can be limited to the case where punctures occur in the upper surface of the water level. In a real situation, the puncture will not be flat. However, due to the limitation of the device, it could not be tested under such conditions. This must be covered in a future study to confirm the suitability of the device to various situations. In addition, in the attachment test conducted during sailing, the ship speed that the magnetic liquid stop device can endure may be different when, in addition to the ship speed, strong waves impact the hull during bad weather at sea.

The results of the test for attachability on round surfaces showed that the magnetic liquid stop device can be effectively applied to curved pipelines or cylinders. However, based on the angle at which each size of the device can be bent according to Equation (3), it was determined that WPP-L can be attached to a diameter of $708 \mathrm{~mm}$ or higher, WPP-M can be attached to a diameter of $498 \mathrm{~mm}$ 
or higher, and WPP-S can be attached to a diameter of $290 \mathrm{~mm}$ or higher. Although the applicability of the round surfaces could be confirmed through the test, there are still limitations with regard to application in practical cases, since the surface of the puncture occurring in an actual ship will often be irregular. This limitation of the study will be overcome by researching the development of highly flexible and stronger devices in the future.

Regarding the magnetic field test, although there was some difference between the theoretically calculated and measured results, it was confirmed that the magnetic field has no influence when it is more than $750 \mathrm{~mm}$ away from the magnet and device. Therefore, it was confirmed that the device has an almost negligible effect on the ship's electronic equipment. This showed the appropriateness of applying more powerful magnets to increase magnetic holding power. Based on this, it is necessary to conduct a study applying a more powerful magnet.

The static electricity test confirmed that the magnetic liquid stop devices did not generate sparks because the magnets were coated with rubber. Although the absence of static electricity was confirmed, this test was limited to examining whether sparks were generated upon contact with the ship's hull or steel plate when the device was used.

The device is considered cost effective if at least 0.33 tonnes of oil spill can be prevented by the large, 0.18 tonnes of oil spill can be prevented by the medium, and 0.13 tonnes of oil spill can be prevented by the small magnetic liquid stop devices used in this experiment. These values were obtained by applying an $\mathrm{F}$ factor of 1.5 according to the recommendation in the previous study. In principle, the $\mathrm{F}$ factor depends on clean-up costs, environmental damages, and socioeconomic costs, which may result in a different $\mathrm{F}$ factor. Therefore, this study has the limitation that different $\mathrm{F}$ factors may be applied depending on the situation because it is determined according to the above-mentioned costs.

In addition, for the magnet to stop the puncture in the hull effectively, the adhesive force of the magnets must be above a certain level. However, a strong adhesive force also makes detachment and attachment difficult. In other words, some difficulty may be experienced if the device must be repositioned in the process of blocking the puncture. This challenge was identified during the testing process, and a handle was attached to the magnetic liquid stop device to act as a lever and enable better detachment and attachment of the device even with its powerful permanent magnets.

Accordingly, this empirical study partly confirmed the practical use of emergency magnetic liquid stop devices. Therefore, the magnetic liquid stop device used in this experiment-based study partially improved problems identified in previous studies, such as slow and ineffective application, applicability to the ship's bottom, attachment to round surfaces, and attachment during sailing. In addition, the safety issues that need to be considered when using the device were verified through spark and magnetic field tests.

Although some usability has been confirmed through empirical studies, it has been recognized that these devices need to be studied and developed by focusing on minimizing the amount of oil spillage that would have occurred in ships, rather than preventing 100\% of liquid spills. Blocking $100 \%$ of an oil spill is physically very challenging and can limit the cost-benefit. Additional studies should be conducted based on this understanding gained from this study and the limitations mentioned above. In addition, although the usability of the device to some curved surfaces was confirmed, it is necessary to develop and experimentally study a device with a more flexible material and a magnet with strong holding force to confirm the applicability of the device to irregular surfaces. In this experimental study, a water tank with a sufficiently large capacity should be developed, and punctures at different heights should be introduced to test the performance of the device under different water pressures. In addition, because no standardized procedure is available for testing this type of devices, it is necessary to propose a standardized procedure based on additional experimental studies. Finally, a verification study should be performed using an actual application prototype of the device. 


\section{Conclusions}

In this study, an emergency, waterproof, magnetic-type oil spill stop device for ships was investigated to improve the limitations identified in earlier studies, such as poor pressure resistance, inadequate attachment of the device, interference from their magnetic fields, potential to generate sparks, and cost effectiveness. Since this study had limitations in applying standardized test methods, it is important to note that this study focused on the verification of practicality of the device.

This experimental study confirmed that the magnetic liquid stop emergency device considered is generally effective for depths of up to approximately $9.8 \mathrm{~m}$ in water. Moreover, the maintenance of the device's attachment during sailing confirmed it to be effective at a ship speed of $18 \mathrm{kts}$. Furthermore, a test was performed to verify the device's attachment to the round surfaces of pipelines and cylindrical vessels. Although limitations were found depending on the device size and the diameter of the round surface, it was found that the device is generally applicable to flat round surfaces. Furthermore, tests were conducted to investigate the effects of magnetic fields and the generation of sparks, which revealed that the device does not affect the navigation and electronic equipment of the ship when located at distances of $750 \mathrm{~mm}$ or higher. Further, a simple free-drop spark test indicated that static electricity was not generated. This experimental study demonstrated that the magnetic liquid stop device is a cost-effective option that can be used to quickly stop the inflow or outflow of liquid in the event of a hull puncture accident.

For the application of this device to an actual ship, the development and application of a more precise and effective device is required to consider the limitations identified in the discussion section. Additional research should be performed to address this.

Author Contributions: C.-J.C. conceived and designed the experiments; C.-J.C. participated and performed the experiments; C.-J.C. analyzed the data; C.-J.C. wrote the paper. The author has read and agreed to the published version of the manuscript.

Funding: This research received no external funding.

Acknowledgments: I would like to express my sincere gratitude to Ki-Seng Kwon, Jung-Hwan Moon, Kyung-Tae Jung, Jin-Kyeong Kim, and Jong-Huwi Yun who gave me motivation of further study regarding liquid spill stopper through their article. I also would like to express my sincere gratitude to Korea Ocean Tech., KIMFT and Korea Coast Guard for their assistance in performing this study.

Conflicts of Interest: The author declares no conflict of interest.

\section{References}

1. Ventikos, N.P.; Psaraftis, H.N. Spill accident modeling: A critical survey of the event-decision network in the context of IMO's formal safety assessment. J. Hazard. Mater. 2004, 107, 59-66. [CrossRef]

2. Su, D.-T.; Tzu, F.-M.; Cheng, C.-H. Investigation of Oil Spills from Oil Tankers through Grey Theory: Events from 1974 to 2016. J. Mar. Sci. Eng. 2019, 7, 373. [CrossRef]

3. Nagelmackers, H.-G. Aftermath of the Amoco Cadiz: Why must the European community act? Mar. Policy 1980, 4, 3-18. [CrossRef]

4. Ketkar, K.W. The Oil Pollution Act of 1990: A Decade Later. Spill Sci. Technol. Bull. 2002, 7, 45-52. [CrossRef]

5. Stenman, C. The Development of the MARPOL and EU Regulations to Phase out Single Hulled Oil Tankers. Master's Thesis, School of Economics and Commercial Law, Göteborg University, Göteborg, Sweden, 2005.

6. Lee, K.-H.; Kim, T.-G.; Cho, Y.-H. Influence of Tidal Current, Wind, and Wave in Hebei Spirit Oil Spill Modeling. J. Mar. Sci. Eng. 2020, 8, 69. [CrossRef]

7. Lee, S.-M.; Ha, M.; Kim, E.-J.; Jeong, W.-C.; Hur, J.; Park, S.G.; Kwon, H.; Hong, Y.-C.; Ha, E.-H.; Lee, J.S.; et al. The effects of wearing protective devices among residents and volunteers participating in the cleanup of the Hebei Spirit oil spill. J. Prev. Med. Public Health 2009, 42, 89-95. [CrossRef] [PubMed]

8. Uri, J. Patch System for Ship Hulls. U.S. Patent 5,036,786 A, 6 August 1991.

9. Lopes, B.V.; Pavlovic, A.; Trombetta, T.B.; Oleinik, P.H.; Monteiro, C.B.; Guimarães, R.C.; Da Silva, D.V.; Marques, W. Numerical Study of Oil Spill in the Patos Lagoon Under Flood and Ebb Conditions. J. Mar. Sci. Eng. 2019, 7, 4. [CrossRef] 
10. Hammoud, B.; Ndagijimana, F.; Faour, G.; Alyad, H.; Jomaah, J. Bayesian statistics of wide-band radar reflections for oil spill detection on rough ocean surface. J. Mar. Sci. Eng. 2019, 7, 12. [CrossRef]

11. Zanier, G.; Palma, M.; Petronio, A.; Roman, F.; Armenio, V. Oil Spill Scenarios in the Kotor Bay: Results from High Resolution Numerical Simulations. J. Mar. Sci. Eng. 2019, 7, 54. [CrossRef]

12. Huang, K.; Nie, W.; Luo, N. A Method of Constructing Marine Oil Spill Scenarios from Flat Text Based on Semantic Analysis. Int. J. Environ. Res. Public Health 2020, 17, 2659. [CrossRef] [PubMed]

13. ITOPF. Oil Tanker Spill Statistics 2019. Available online: https://www.itopf.org/knowledge-resources/datastatistics/statistics/ (accessed on 10 May 2020).

14. IMO. The Guidelines for Formal Safety Assessment (FSA) for Use in the IMO Rule-Making Process; MSC/Circ.1023/MEPC/Circ.392; IMO: London, UK, 2002.

15. IMO. Formal Safety Assessment, Liquefied Natural Gas (LNG) Carriers; MSC 83/21/1; IMO: London, UK, 2007.

16. IMO. Formal Safety Assessment, Container Vessels; MSC 83/21/2; IMO: London, UK, 2007.

17. IMO. Formal Safety Assessment, Cruise Ships; MSC 85/17/1; MSC 85/INF.2; IMO: London, UK, 2008.

18. IMO. Formal Safety Assessment, Crude Oil Tankers; MEPC 58/17/2; MEPC 58/INF. 2; IMO: London, UK, 2008.

19. Kontovas, C.A.; Psaraftis, H.N. Formal Safety Assessment: A Critical Review and Ways to Strengthen It and Make It More Transparent. In Proceedings of the NTUA-MT-06-102 Meeting; The National Technical University of Athens: TAthens, Greece, 2020. Available online: http://martrans.org/documents/2006/safety/Kontovas\% 20Psaraftis\%20FSA\%20FINAL\%20single\%20spaced.pdf (accessed on 10 May 2020).

20. Frisbie, J.F. Apparatus for Preventing Ships from Sinking. U.S. Patent 351,971 A, 2 November 1886.

21. Weihe, L. Leak-Stopper for Vessels. U.S. Patent 381,302 A, 17 April 1888.

22. Richrdson, J. Leak Stopper for Ships. U.S. Patent 385,572 A, 3 July 1888.

23. Buce, W.F. Apparatus and Method for Sealing a Vessel Opening. U.S. Patent 3,669,055 A, 13 June 1972.

24. McDuff, G.L.; McCoy, B.G. Seal Pac Diaper. U.S. Patent 4,712,502 A, 15 December 1987.

25. Johnson, R.F. Oil Spill Control. U.S. Patent 5,009,179 A, 23 April 1991.

26. Krueger, L. Magnetic Curtain for Covering Leaks in Ships. U.S. Patent 770,078 A, 13 September 1904.

27. Williams, W.E. Magnetic Ship Hull Patch. U.S. Patent 5,165,356 A, 24 November 1992.

28. Prysner, W.J. Device for Reducing Flow of Fluid from a Ruptured Vessel. U.S. Patent 5,685,252 A, 11 November 1997.

29. Cunningham, C.C.; Gaudreau, M.P.; Sigmar, A.; Hogan, E.M. Sealing Openings in Hulls of Vessels. U.S. Patent 5,782,196 A, 21 July 1998.

30. Del Raso, A. Emergency Bulk Liquid Cargo Spill Prevention System. U.S. Patent 6,152,059 A, 28 November 2000.

31. Moon, J.-H.; Kim, J.-K.; Kwon, K.-S.; Jung, K.-T.; Yun, J.-H. An Experimental Study on the Performance of Liquid Spill Stopper to Prevent Flood through the Hull Punctures. J. Navig. Port. Res. 2013, 37, 149-154. [CrossRef]

32. Berg, T.; Kvamstad, B.; Kjersem, F. Safety at Sea-A Review of Norwegian Activities. TransNav 2011, 5, 195-201.

33. Marine, M. Magnetic Miko Plaster. 2019. Available online: https://mikomarine.com/underwater-blankingtools/magnetic-miko-plaster/ (accessed on 10 December 2019).

34. Lin, Y. Magnetic Properties of Hot Deformed and Boundary Diffused Nd-Fe-B Permanent Magnet; University of Nebraska: Lincoln, NE, USA, 2017; pp. 1-75.

35. Man, H.H.; Man, H.C.; Leung, L.K. Corrosion protection of NdFeB magnets by surface coatings-Part I: Salt spray test. J. Magn. Magn. Mater. 1996, 152, 40-46. [CrossRef]

36. KOT. Water Proof Equipment; KOT: Busan, Korea, 2017.

37. KOT. KOT-LSS-DLM; KOT: Busan, Korea, 2017.

38. KOT. Magnetic Type Liquid Stop Device. 2019. Available online: http://www.koreaot.co.kr/?page=move\& flag=user\&type=product (accessed on 15 July 2019).

39. ASME. ASME Boiler \& Pressure Vessel Code. In Rules for Construction of Pressure Vessels; The American Society of Mechanical Engineers: New York, NY, USA, 2019.

40. White, F.M. Fluid Mechanics, 7th ed.; McGraw-Hill: New York, NY, USA, 2011.

41. Supermagnete. How Do You Calculate the Magnetic Flux Density. Available online: https://www.supermagnete.de/eng/faq/How-do-you-calculate-the-magnetic-flux-density\#formulafor-block-magnet-flux-density (accessed on 5 November 2019). 
42. Magnetics, A. Flux Calculator. Available online: https://magnet.com.au/magnet-strength-flux-calculator.html (accessed on 10 September 2019).

43. Koppel, T.; Tasa, T.; Tint, P. Electromagnetic fields in contemporary office workplaces. Agron. Res. 2013, 11, 421-434.

44. International Commission on Non-Ionizing Radiation Protection. Guidelines for limiting exposure to electric fields induced by movement of the human body in a static magnetic field and by time-varying magnetic fields below $1 \mathrm{~Hz}$. Health Phys. 2014, 106, 418-425. [CrossRef] [PubMed]

45. MAGNA. For the Magnet Furture MAGNA Co., Ltd. Available online: https://www.magna-tokyo.com/eng/ kensasokutei (accessed on 10 April 2020).

46. Uğurlu, Ö. Analysis of fire and explosion accidents occurring in tankers transporting hazardous cargoes. Int. J. Ind. Ergon. 2016, 55, 1-11. [CrossRef]

47. IMO. Revised Guidelines for Formal Safety Assessment (FSA) for Use in the IMO Rule-Making Process; IMO: London, UK, 2015.

48. IMO. Revised Guidelines for Formal Safety Assessment (FSA) for Use in the IMO Rule-Making Process; MSC-MEPC.2/Circ.12/Rev.2; IMO: London, UK, 2018.

49. Vanem, E.; Endresen, Ø.; Skjong, R. Cost-effectiveness criteria for marine oil spill preventive measures. Reliab. Eng. Syst. Saf. 2008, 93, 1354-1368. [CrossRef]

50. Macrotrends. Crude Oil Prices-70 Year Historical Chart. 2020. Available online: https://www.macrotrends. net/1369/crude-oil-price-history-chart (accessed on 10 February 2020).

(C) 2020 by the author. Licensee MDPI, Basel, Switzerland. This article is an open access article distributed under the terms and conditions of the Creative Commons Attribution (CC BY) license (http://creativecommons.org/licenses/by/4.0/). 